J. Jpn. Soc. Comp. Statist., 18(2005), 61-73

\title{
SENSITIVITY ANALYSIS IN FUNCTIONAL REGRESSION MODELS FOR SCALAR RESPONSES
}

\author{
Norihiro Harasawa, Kaoru Fueda ${ }^{\dagger}$ and Yutaka Tanaka ${ }^{\ddagger}$
}

\begin{abstract}
In this paper, we propose a method of sensitivity analysis in functional regression models for scalar responses. We define a Cook's D type distance in functional regression analysis (FRA) based on two kinds of influence functions: 1) Empirical Influence Function (EIF), 2) Sample Influence function (SIF). In ordinary regression analysis (ORA), the Cook's D distance can be expressed as a function of residual and leverage. We define diagnostic statistics which correspond to residual and leverage in ORA, and show our Cook's D type distances in FRA are functions of these diagnostic statistics. We give a numerical example to show the properties of two types of Cook's D type distance and these diagnostic statistics.
\end{abstract}

\section{Introduction}

The general model building process consists of 1) identification of an appropriate model, 2) estimating parameters contained in the model, 3) diagnostic checking and 4) interpretation of the result of analysis (Box and Jenkins, 1970). If any inadequacy is found, we have to go back to step 1. Among these four steps, the third step plays an important role. Methods of diagnostic checking have been developed at first in regressions and related methods (see, e.g., Cook and Weisberg, 1982; Chatterjee and Hadi, 1988) and then expanded into other statistical models. However few studies have been carried out in the field of functional data analysis.

Functional data analysis is composed of various methods such as functional regression analysis, functional principal components analysis and functional canonical correlation analysis to analyze the curves or functions (Ramsay, 1982; Ramsay and Dalzell, 1991; Ramsay and Silverman, 1997). Among them functional regression analysis enables us to investigate the pattern between response and explanatory variable functions over time or some other arguments.

Yamanishi and Tanaka (2005) proposed a method of sensitivity analysis in functional principal component analysis. They derived influence functions for eigenvalues and eigenfunctions with and without taking account of the effect through the influence on the smoothing parameters. Then they defined influence statistics to detect influential observations.

In this paper, we propose a method of sensitivity analysis to investigate the effects of influential observations on the regression coefficient functions in functional regression analysis. It is known in ordinary regression analysis that Cook's D and similar influence measures

\footnotetext{
*Graduate School of Natural Science and Technology, Okayama University, 3-1-1, Tsushima-naka, Okayama 700-8530, Japan E-mail: nohi@f7.ems.okayama-u.ac.jp

†Graduate School of Environmental Science, Okayama University, 2-1-1, Tsushima-naka, Okayama 7000530, Japan

${ }^{\ddagger}$ Department of Mathematical Sciences, Nanzan University, 27, Seirei cho, Seto, Nagoya 489-0863, Japan

Key words: Cook's distance; Functional data; Influence function
} 
consist of two components, residual and leverage, and these components provide useful information of explaining the reason of large influence. We propose Cook's D type influence measures in functional regression analysis and try to decompose them into components which provide additional information on the reason as in the case of ordinary regression analysis.

In Section 2, we introduce functional regression analysis for scalar responses and in Section 3, we propose a method of sensitivity analysis in functional regression analysis and show a numerical example. In Section 4, we provide our concluding remarks.

\section{Functional regression model for scalar responses}

A functional regression model for scalar response is defined as follows (Ramsay and Silverman, 1997; Shimokawa, Mizuta and Sato, 2000):

$$
y_{i}=\beta_{0}+\sum_{g=1}^{G} \int x_{i g}(s) \beta_{g}(s) d s+\epsilon_{i} \quad(i=1, \ldots, n),
$$

where $x_{i g}(s)$ is the $i$-th observation of the $g$-th functional covariate, $\beta_{0}$ is the intercept, $G$ is the number of functional covariates, $\beta_{g}(s)$ is the regression coefficient function for the $g$-th functional covariate, $\epsilon_{i}$ is the error term, and $n$ is the number of observations. We use the integrated squared second derivative $P E N\left(\beta_{g}(s)\right)=\int\left(D^{2} \beta_{g}(s) / D s^{2}\right)^{2} d s$ as the roughness penalty.

\subsection{Parametric model and parameter estimation}

To estimate the regression coefficient functions $\beta_{g}(s)$, we consider a class of functions

$$
\left\{\left(\beta_{1}(s), \ldots, \beta_{G}(s)\right) \mid \beta_{g}(s)=\sum_{j=1}^{K_{g}} b_{j g} \phi_{j g}(s)=\mathbf{b}_{g}^{\mathrm{T}} \Phi_{g}(s), g=1, \ldots, G\right\},
$$

where $\mathbf{b}_{g}=\left(b_{1 g}, \ldots, b_{K_{g} g}\right)^{\mathrm{T}}, \Phi_{g}(s)=\left(\phi_{1 g}(s), \ldots, \phi_{K_{g} g}(s)\right)^{\mathrm{T}}, g=1, \ldots, G$, and $\left\{\phi_{j g}(s), g=\right.$ $\left.1, \ldots, K_{g}\right\}$ are basis functions and $K_{g}$ is the number of basis functions. The penalized least squares estimator of $\left(\beta_{1}(s), \ldots, \beta_{G}(s)\right)$ is given by minimizing the following penalized integrated sum of squared error:

$$
P E N S S E=\sum_{i=1}^{n}\left(y_{i}-\left(\beta_{0}+\sum_{g=1}^{G} \int x_{i g}(s) \beta_{g}(s) d s\right)\right)^{2}+\sum_{g=1}^{G} \lambda_{g} P E N\left(\beta_{g}(s)\right)
$$

The balance between goodness of fit and roughness of the function $\beta_{g}(s)$ is controlled by the smoothing parameters $\lambda_{g}$.

Now consider estimation of the parameter coefficients $b_{j g}, g=1, \ldots, G, j=1, \ldots, K_{g}$ for fixed $\lambda$ and $K_{g}$. We at first expand the covariate functions as

$$
x_{i g}(s)=\sum_{j=1}^{K_{g}} c_{i j g} \phi_{j g}(s)+\epsilon_{i g}(s)=\mathbf{c}_{i g}^{\mathrm{T}} \Phi_{g}(s)+\epsilon_{i g}(s),
$$

and obtain the coefficients $c_{i j}$, where $\epsilon_{i g}(s)$ is a truncation error which satisfies $\int \phi_{j g}(s) \epsilon_{i g}(s) d s=0, g=1, \ldots, G, i=1, \ldots, n$. Then the criterion (1) is represented 
as the following matrix equation:

$$
P E N S S E=\left(\mathbf{y}-\mathbf{Z} \mathbf{b}^{*}\right)^{\mathrm{T}}\left(\mathbf{y}-\mathbf{Z} \mathbf{b}^{*}\right)+\sum_{g=1}^{G} \lambda_{g} \mathbf{b}_{g}^{\mathrm{T}} \mathbf{R}_{g} \mathbf{b}_{g}
$$

where

$$
\begin{aligned}
\mathbf{J}_{g} & =\int \Phi_{g}(s) \Phi_{g}^{\mathrm{T}}(s) d s, \quad \mathbf{C}_{g}=\left(\mathbf{c}_{1 g}\left(K_{g}\right), \ldots, \mathbf{c}_{n g}\left(K_{g}\right)\right)^{\mathrm{T}} \\
\mathbf{Z} & =\left(\mathbf{1}, \mathbf{C}_{1} \mathbf{J}_{1}, \ldots, \mathbf{C}_{G} \mathbf{J}_{G}\right)=\left(\mathbf{z}_{1}, \ldots, \mathbf{z}_{n}\right)^{\mathrm{T}}, \quad \mathbf{b}^{*}=\left(\beta_{0}, \mathbf{b}_{1}^{\mathrm{T}}, \ldots, \mathbf{b}_{G}^{\mathrm{T}}\right)^{\mathrm{T}}, \\
\mathbf{R}_{g} & =\int\left(D^{2} \Phi_{g}(s)\right)\left(D^{2} \Phi_{g}(s)\right)^{\mathrm{T}} d s \quad \text { and } \mathbf{y}=\left(y_{1}, \ldots, y_{n}\right)^{\mathrm{T}} .
\end{aligned}
$$

In the above, it is described as though the functional covariates $\left\{x_{i g}(s)\right\}$ are observed. When they are observed only at sampled points, we have to transform in advance the observed values to functional covariates by using an appropriate smoothing method.

\subsection{Choice of smoothing parameters and the numbers of basis functions}

The basis functions and smoothing parameters are very important in functional data analysis. There are various types of basis such as Fourier series, polynomial basis, spline basis and radial basis. In using such bases, we have to choose the numbers of basis functions. Green and Silverman (1994), Simonoff (1996) used a cross-validation method to choose a smoothing parameter $\lambda$ and Ando, Imoto and Konish (2001) proposed Generalized Information Criteria to choose parameters $\left\{\lambda_{g}\right\}$ and the numbers of basis functions $\left\{K_{g}\right\}$. In this paper, we use a cross-validation method to choose $\left\{\lambda_{g}\right\}$ and $\left\{K_{g}\right\}$.

Let $\mathbf{b}^{*[-i]}(\mathbf{K}, \lambda)$ be the minimizer of

$$
\left(\mathbf{y}^{[-i]}-\mathbf{Z}^{[-i]} \mathbf{b}^{*}\right)^{\mathrm{T}}\left(\mathbf{y}^{[-i]}-\mathbf{Z}^{[-i]} \mathbf{b}^{*}\right)+\sum_{g=1}^{G} \lambda_{g} \mathbf{b}_{g}^{\mathrm{T}} \mathbf{R}_{g} \mathbf{b}_{g}
$$

for fixed $\mathbf{K}=\left(K_{1}, \ldots, K_{G}\right)$ and $\lambda=\left(\lambda_{1}, \ldots, \lambda_{G}\right)$, where

$$
\begin{aligned}
& \mathbf{y}^{[-i]}=\left(y_{1}, \ldots, y_{i-1}, y_{i+1}, \ldots, y_{n}\right), \\
& \mathbf{Z}^{[-i]}=\left(\mathbf{z}_{1}, \ldots, \mathbf{z}_{i-1}, \mathbf{z}_{i+1}, \ldots, \mathbf{z}_{n}\right),
\end{aligned}
$$

Then the cross-validated value

$$
C V(\mathbf{K}, \lambda)=\sum_{i=1}^{n}\left(y_{i}-\mathbf{z}_{i} \mathbf{b}^{*[-i]}(\mathbf{K}, \lambda)\right)^{2},
$$

depends on $\mathbf{K}$ and $\lambda$. We select $\mathbf{K}$ and $\lambda$ which minimize $C V(\mathbf{K}, \lambda)$. For selected $\mathbf{K}$ and $\lambda$, we have the minimizer of (2) as $\hat{\mathbf{b}}^{*}=\left(\mathbf{Z}^{\mathrm{T}} \mathbf{Z}+\Lambda\right)^{-1} \mathbf{Z}^{\mathrm{T}} \mathbf{y}$, where $\Lambda=\operatorname{diag}\left(0, \lambda_{1} \mathbf{R}_{1}, \ldots, \lambda_{G} \mathbf{R}_{G}\right)$ and the predicted values $\hat{\mathbf{y}}$ corresponding to the observed values $\mathbf{y}$ are given by

$$
\hat{\mathbf{y}}=\mathbf{Z}\left(\mathbf{Z}^{\mathrm{T}} \mathbf{Z}+\Lambda\right)^{-1} \mathbf{Z}^{\mathrm{T}} \mathbf{y} .
$$

$\mathbf{P}=\mathbf{Z}\left(\mathbf{Z}^{\mathrm{T}} \mathbf{Z}+\Lambda\right)^{-1} \mathbf{Z}^{\mathrm{T}}$ plays a role like the hat matrix in ordinary regression analysis (ORA). We call the $(i, i)$-th element of $\mathbf{P}$,

$$
p_{i i}=\mathbf{z}_{i}^{\mathrm{T}}\left(\mathbf{Z}^{\mathrm{T}} \mathbf{Z}+\Lambda\right)^{-1} \mathbf{z}_{i}
$$


the leverage of individual $i$ for the penalized model, while we call the $(i, i)$-th element of $\mathbf{H}=\mathbf{Z}\left(\mathbf{Z}^{\mathrm{T}} \mathbf{Z}\right)^{-} \mathbf{Z}^{\mathrm{T}}$,

$$
h_{i i}=\mathbf{z}_{i}^{\mathrm{T}}\left(\mathbf{Z}^{\mathrm{T}} \mathbf{Z}\right)^{-} \mathbf{z}_{i}
$$

the leverage of individual $i$ for the non-penalized model, where superscript ${ }^{-}$indicates the generalized inverse.

\section{Sensitivity analysis in functional regression analysis}

\subsection{Influence function for regression coefficient function}

Sensitivity analysis is well-established in many statistical methods to explore whether there exist influential observations and to detect such observations, if any. To evaluate the influence of each individual on the result of the analysis we make use of the idea of an influence function (Hampel, 1974). To do this we introduce case-weight $w_{i}^{*}=n w_{i} / \sum_{j=1}^{n} w_{j}$ for each individual $i$ and perturb the case-weights from $\mathbf{w}_{0}=\left\{w_{i}=1, i=1, \ldots, n\right\}$ to $\mathbf{w}=\left\{w_{i}, i=1, \ldots, n\right\}$.

Based on the fact that $n$ times the partial derivative of $\hat{\mathbf{b}}_{g}$ with respect to $w_{i}$ is equal to the empirical influence function $\operatorname{EIF}\left(\mathbf{z}_{i} ; \hat{\mathbf{b}}_{g}\right.$ ) (Tanaka and Zhang, 1999), we define the EIF of $\hat{\boldsymbol{\beta}}(s)=\left(\hat{\beta}_{0}(s), \ldots, \hat{\boldsymbol{\beta}}_{G}(s)\right)^{\mathrm{T}}$, the coefficient function to $\boldsymbol{x}_{i}(s)=\left(x_{i 1}(s), \ldots, x_{i G}(s)\right)^{\mathrm{T}}$, by

$$
\operatorname{EIF}\left(\boldsymbol{x}_{i}(s) ; \hat{\boldsymbol{\beta}}(s)\right)=\left.\frac{\partial \hat{\boldsymbol{\beta}}(s)}{\partial w_{i}}\right|_{\mathbf{w}_{0}} .
$$

We also define the sample influence function (SIF) by

$$
S I F\left(\boldsymbol{x}_{i}(s) ; \hat{\boldsymbol{\beta}}(s)\right)=-\left(\hat{\boldsymbol{\beta}}^{[-i]}(s)-\hat{\boldsymbol{\beta}}(s)\right) .
$$

The penalized least squares criterion PENSSE with case-weight $\mathbf{W}=\operatorname{diag}\left(n w_{1} / \sum_{j=1}^{n} w_{j}\right.$, $\left.\ldots, n w_{n} / \sum_{j=1}^{n} w_{j}\right)$ can be given by

$$
P E N S S E=\left(\mathbf{y}-\mathbf{Z} \mathbf{b}^{*}\right)^{\mathrm{T}} \mathbf{W}\left(\mathbf{y}-\mathbf{Z} \mathbf{b}^{*}\right)+\sum_{g=1}^{G} \lambda_{g} \mathbf{b}_{g}^{\mathrm{T}} \mathbf{R}_{g} \mathbf{b}_{g},
$$

and $\left\{\hat{\mathbf{b}}_{g}\right\}$, which minimize PENSSE, are obtained by solving the normal equation in the form

$$
\left(\mathbf{Z}^{\mathrm{T}} \mathbf{W} \mathbf{Z}+\Lambda\right) \hat{\mathbf{b}}^{*}=\mathbf{Z}^{\mathrm{T}} \mathbf{W} \mathbf{y}
$$

\subsection{Influence function}

For fixed $\mathbf{K}$ and $\lambda$, the partial derivative of (4) with respect to $w_{i}$ leads to the following equation.

$$
\left(\mathbf{Z}^{\mathrm{T}} \frac{\partial \mathbf{W}}{\partial w_{i}} \mathbf{Z}\right) \hat{\mathbf{b}}^{*}+\left(\mathbf{Z}^{\mathrm{T}} \mathbf{W} \mathbf{Z}+\Lambda\right) \frac{\partial \hat{\mathbf{b}}^{*}}{\partial w_{i}}=\mathbf{Z}^{\mathrm{T}} \frac{\partial \mathbf{W}}{\partial w_{i}} \mathbf{y}
$$


Then, we have the EIF of coefficient function $\hat{\boldsymbol{\beta}}(s)$ by

$$
\begin{array}{r}
\left.\frac{\partial \hat{\boldsymbol{\beta}}(s)}{\partial w_{i}}\right|_{\mathbf{w}_{0}}=\Phi^{*}(s)\left(\left.\frac{\partial \hat{\mathbf{b}}^{*}}{\partial w_{i}}\right|_{\mathbf{w}_{0}}\right)=\Phi^{*}(s)\left\{\left(\mathbf{Z}^{\mathrm{T}} \mathbf{Z}+\Lambda\right)^{-1}\left(\mathbf{z}_{i} r_{i}-\overline{\mathbf{z} r}\right)\right\} \\
\text { where } \Phi^{*}(s)=\left(\begin{array}{ccc}
1 & & \mathbf{0} \\
& \Phi_{1}(s) & \\
& \ddots & \\
& & \\
\mathbf{0} & & \Phi_{G}(s)
\end{array}\right),
\end{array}
$$

$r_{i}=y_{i}-\mathbf{z}_{i}^{\mathrm{T}} \hat{\mathbf{b}}^{*}$ and $\overline{\mathbf{z} r}=n^{-1} \sum_{i=1}^{n} \mathbf{z}_{i} r_{i}$. We also have the SIF of $\hat{\boldsymbol{\beta}}(s)$ by

$$
-\left(\hat{\boldsymbol{\beta}}^{[-i]}(s)-\hat{\boldsymbol{\beta}}(s)\right)=-\Phi^{*}(s)\left(\hat{\mathbf{b}}^{*[-i]}-\hat{\mathbf{b}}^{*}\right)=\Phi^{*}(s)\left(1-p_{i i}\right)^{-1}\left\{\left(\mathbf{Z}^{\mathrm{T}} \mathbf{Z}+\Lambda\right)^{-1} \mathbf{z}_{i} r_{i}\right\} .
$$

The proportion of $(5)$ to $(6)$ is $S I F / E I F \approx 1-p_{i i}$. This relation is similar to that in the case of ORA, where $S I F / E I F=1-h_{i i}$.

\subsection{Cook's D type distance based on EIF}

To detect singly influential observations, we use Cook's D type statistic, which we simply call Cook's D. Let $D_{E P i}$ be Cook's D based on EIF, which is given by

$$
\begin{aligned}
D_{E P i} & =\left.\left(\left.\frac{\partial \hat{\mathbf{b}}^{*}}{\partial w_{i}}\right|_{\mathbf{w}_{0}}\right)^{\mathrm{T}}\left(\hat{\operatorname{Cov}}\left(\hat{\mathbf{b}}^{*}\right)\right)^{-} \frac{\partial \hat{\mathbf{b}}^{*}}{\partial w_{i}}\right|_{\mathbf{w}_{0}} \\
& =\left.\left(\left.\frac{\partial \hat{\mathbf{b}}^{*}}{\partial w_{i}}\right|_{\mathbf{w}_{0}}\right)^{\mathrm{T}}\left(\hat{\sigma}^{2}\left(\mathbf{Z}^{\mathrm{T}} \mathbf{Z}+\Lambda\right)^{-1} \mathbf{Z}^{\mathrm{T}} \mathbf{Z}\left(\mathbf{Z}^{\mathrm{T}} \mathbf{Z}+\Lambda\right)^{-1}\right)^{-} \frac{\partial \hat{\mathbf{b}}^{*}}{\partial w_{i}}\right|_{\mathbf{w}_{0}} .
\end{aligned}
$$

Here, we use the property $\left(P_{1} A P_{2}\right)^{-}=P_{2}^{-1} A^{-} P_{1}^{-1}$ in the case where $P_{1}, P_{2}$ are nonsingular (Pringle and Rayner, 1971), then we can rewrite as

$$
\left[\left(\mathbf{Z}^{\mathrm{T}} \mathbf{Z}+\Lambda\right)^{-1} \mathbf{Z}^{\mathrm{T}} \mathbf{Z}\left(\mathbf{Z}^{\mathrm{T}} \mathbf{Z}+\Lambda\right)^{-1}\right]^{-}=\left(\mathbf{Z}^{\mathrm{T}} \mathbf{Z}+\Lambda\right)\left[\mathbf{Z}^{\mathrm{T}} \mathbf{Z}\right]^{-}\left(\mathbf{Z}^{\mathrm{T}} \mathbf{Z}+\Lambda\right)
$$

So, we obtain

$$
\begin{aligned}
D_{E P i} & =\left(\hat{\sigma}^{2}\right)^{-1}\left(\mathbf{z}_{i} r_{i}-\overline{\mathbf{z} r}\right)^{\mathrm{T}}\left(\mathbf{Z}^{\mathrm{T}} \mathbf{Z}\right)^{-}\left(\mathbf{z}_{i} r_{i}-\overline{\mathbf{z} r}\right) \\
& =\left(\hat{\sigma}^{2}\right)^{-1}\left\{h_{i i} r_{i}^{2}-2 r_{i} n^{-1} \sum_{j=1}^{n} h_{i j} r_{j}+n^{-2} \sum_{j=1}^{n} \sum_{k=1}^{n} h_{j k} r_{j} r_{k}\right\},
\end{aligned}
$$

where $\hat{\sigma}^{2}=(n-\operatorname{trace}(\mathbf{P}))^{-1} \sum_{i=1}^{n} r_{i}^{2}$ (Buja, Hastie and Tibshirani, 1989). It can be shown that $D_{E P i}$ is invariant for any generalized inverse. When the second and third terms can be neglected in the last equation in (8), $D_{E P i}$ is approximated by the following equation, which contains the $i$-th residual $r_{i}$ and the $i$-th leverage for the non-penalized model $h_{i i}$.

$$
D_{E P i} \approx\left(\hat{\sigma}^{2}\right)^{-1} h_{i i} r_{i}^{2}
$$




\subsection{Cook's D type distance based on SIF}

Let $D_{S P i}$ be Cook's D based on SIF, which is given by

$$
\begin{aligned}
D_{S P i}= & \left(-\left(\hat{\mathbf{b}}^{*[-i]}-\hat{\mathbf{b}}^{*}\right)\right)^{\mathrm{T}}\left(\hat{\operatorname{Cov}}\left(\hat{\mathbf{b}}^{*}\right)\right)^{-}\left(-\left(\hat{\mathbf{b}}^{*[-i]}-\hat{\mathbf{b}}^{*}\right)\right) \\
= & \left(\hat{\sigma}^{2}\right)^{-1}\left(1-p_{i i}\right)^{-2} r_{i}^{2} \mathbf{z}_{i}^{\mathrm{T}}\left(\mathbf{Z}^{\mathrm{T}} \mathbf{Z}+\Lambda\right)^{-1}\left[\left(\mathbf{Z}^{\mathrm{T}} \mathbf{Z}+\Lambda\right)^{-1} \mathbf{Z}^{\mathrm{T}} \mathbf{Z}\left(\mathbf{Z}^{\mathrm{T}} \mathbf{Z}+\Lambda\right)^{-1}\right]^{-} \\
& \left(\mathbf{Z}^{\mathrm{T}} \mathbf{Z}+\Lambda\right)^{-1} \mathbf{z}_{i} .
\end{aligned}
$$

Using (7), we can derive

$$
\begin{aligned}
D_{S P i} & =\left(\hat{\sigma}^{2}\right)^{-1}\left(1-p_{i i}\right)^{-2} r_{i}^{2} \mathbf{z}_{i}^{\mathrm{T}}\left(\mathbf{Z}^{\mathrm{T}} \mathbf{Z}\right)^{-} \mathbf{z}_{i} \\
& =\left(\hat{\sigma}^{2}\right)^{-1} \frac{h_{i i} r_{i}^{2}}{\left(1-p_{i i}\right)^{2}} .
\end{aligned}
$$

Note that $D_{S P i}$ consists of the residual $r_{i}$, leverage for non-penalized model $h_{i i}$ and leverage for penalized model $p_{i i}$. As shown by Harasawa, Fueda and Tanaka (2004), $D_{S P i}$ is also invariant for any generalized inverse. $D_{S P i}$ can be approximated by

$$
D_{S P i} \approx \frac{D_{E P i}}{\left(1-p_{i i}\right)^{2}} .
$$

\subsection{PCA with metric $\operatorname{Cov}\left(\hat{\mathbf{b}}^{*}\right)^{-}$}

To search for influential subsets of observations Tanaka (1994) suggests applying a "PCA with metric $\operatorname{cov}\left(\hat{\mathbf{b}}^{*}\right)^{-"}$ to the empirical influence function $\left\{E I F\left(\mathbf{z}_{i} ; \hat{\mathbf{b}}^{*}\right)\right\}$, namely to solve a $\Sigma_{g=1}^{G} K_{g} \times \Sigma_{g=1}^{G} K_{g}$ eigenvalue problem.

$$
\left\{n^{-1}[E I F]^{\mathrm{T}}[E I F]-a\left[\hat{\operatorname{Cov}}\left(\hat{\mathbf{b}}^{*}\right)\right]\right\} \mathbf{u}=\mathbf{0},
$$

where $[E I F]$ is a $\Sigma_{g=1}^{G} K_{g} \times n$ matrix of $\left\{E I F\left(\mathbf{z}_{i} ; \hat{\mathbf{b}}^{*}\right)\right\}, a$ is an eigenvalue and $\mathbf{u}$ is a $\Sigma_{g=1}^{G} K_{g} \times 1$ eigenvector.

\subsection{Numerical example}

We analyze the relationship between the rice yields per unit area per year and the measurements of daily temperature in the previous year of the 47 prefectures (Japan Meteorological Agency, 1999; http://www.maff.go.jp/www/info/index.html). The daily temperature data are measured at a weather station in each prefecture and are regarded as functional data.

At first we fit a functional regression model to a dataset of the rice yields and the temperature functions. The data are shown in Figures 1(a) and 1(b). In this study, a Fourier series is used as the basis functions and the number of bases $\mathbf{K}$ and the smoothing parameter $\lambda$ are determined by cross-validation.

Optimal values $\lambda=10^{6}$ and $\mathbf{K}=30$ are determined so that they give the minimum of the cross-validated values. Figure 2 shows the cross-validated values defined in (3) for various values of $\log _{10}(\lambda)$ and $\mathbf{K}$, and Figure 3 shows the plots of the cross sections at $\mathbf{K}=30$ and at $\log _{10}(\lambda)=6$ in Figure 2. The results of functional regression are shown in Figures $4(\mathrm{a})$ and $4(\mathrm{~b})$. The estimated regression coefficient function $\beta_{1}$ is given in Figure 4(a) and the scatter plot of $\mathbf{y}$ and $\hat{\mathbf{y}}$ is given in Figure 4(b). The temperature in February is positively associated with the rice yield and the temperature in March is negatively associated with the rice yield. 


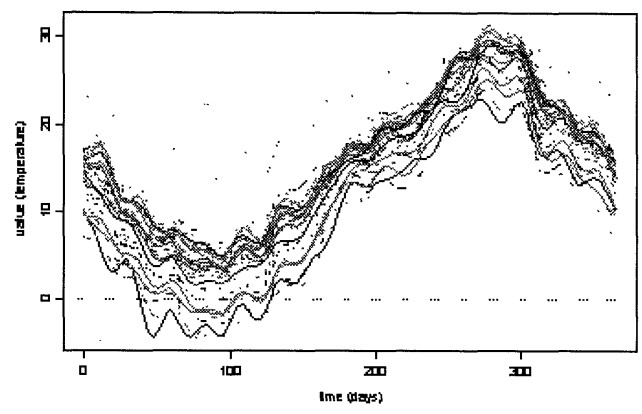

(a)

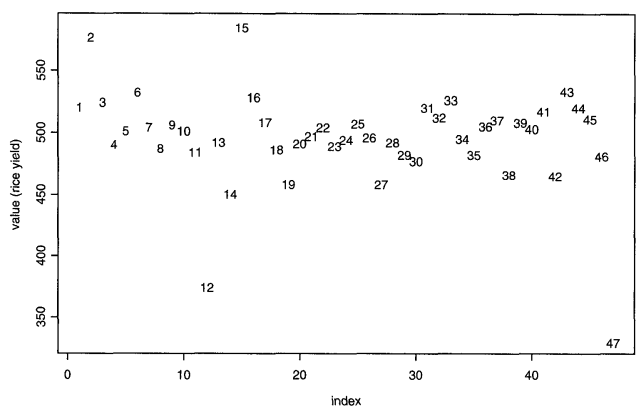

(b)

Fig. 1: Example data: (a) temperature function $x(s)$; (b) rice yield $y$

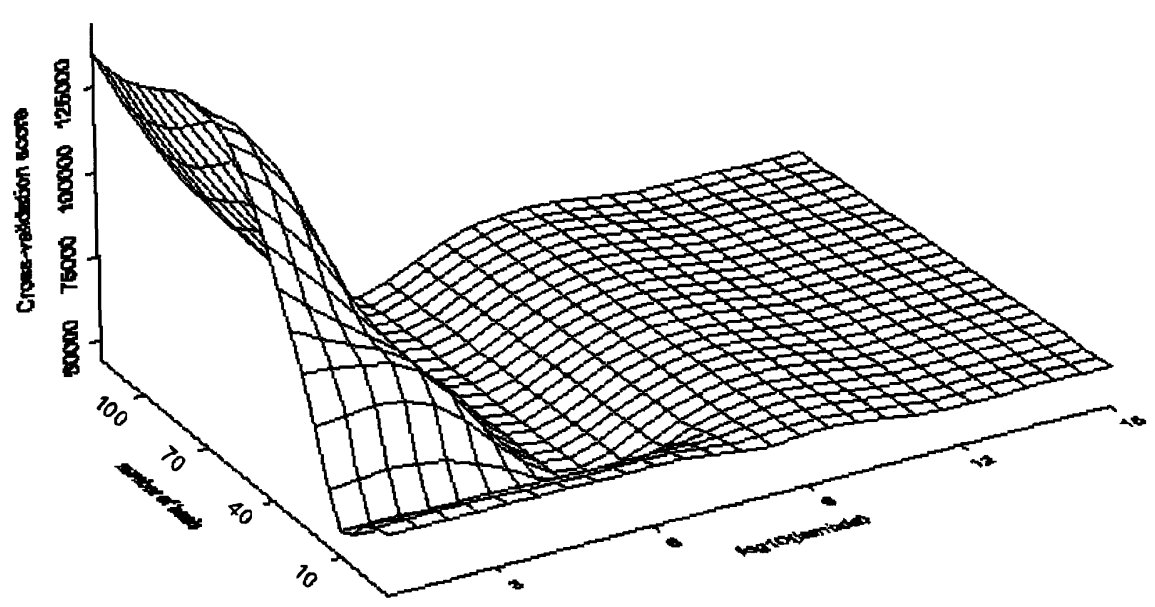

Fig. 2: Surface of cross-validated value against $\log _{10}(\lambda)$ and $\mathbf{K}$

Then we apply our method of sensitivity analysis to check if there exist any influential observations. Cook's Ds based on the EIF and SIF are given in Figure 5(a) and Figure 6(a). Observations No.12 and 4 give the largest and the second largest values of Cook's D in both measures, but the orders of their magnitudes are different. To study their influences in detail in the case we use the EIF the contour plot of Cook's D for $h_{i i}$ and $\left|r_{i}\right|$ are shown in Figure 5 (b). This figure makes clear that the major reason for the large influence of No.12 (Tokyo) is that it has a large residual while that of No.4 (Iwate) is that both the $h_{i i}$ and $r_{i}$ are relatively large. The validity of this discussion is based on the goodness of approximation of (9), but as given in Figure 7, the approximation is good in this example. Similarly the 


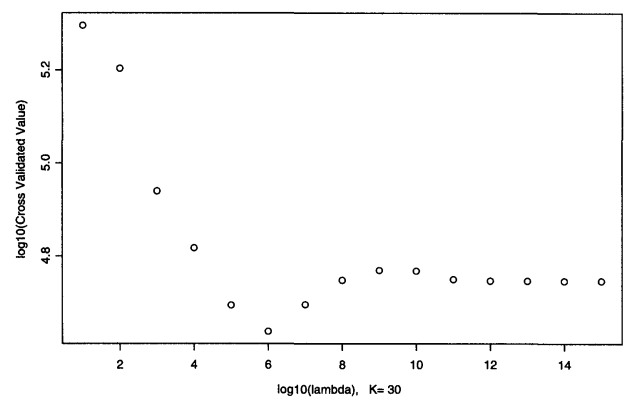

(a)

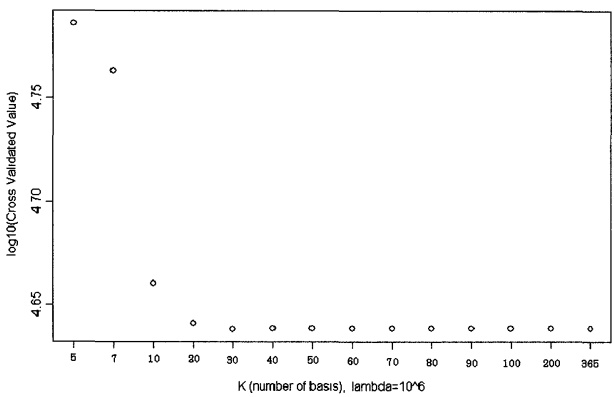

(b)

Fig. 3: Cross section of Figure 2: (a) cross-validated value against $\log _{10}(\lambda)$ at $\mathbf{K}=30$. $x$ axis: $\log _{10}(\lambda), y$-axis: $\log _{10}$ (cross-validated value); (b) cross-validated value against $\mathbf{K}$ at $\log _{10}(\lambda)=6$. $x$-axis: $\mathbf{K}, y$-axis: $\log _{10}$ (cross-validated value)

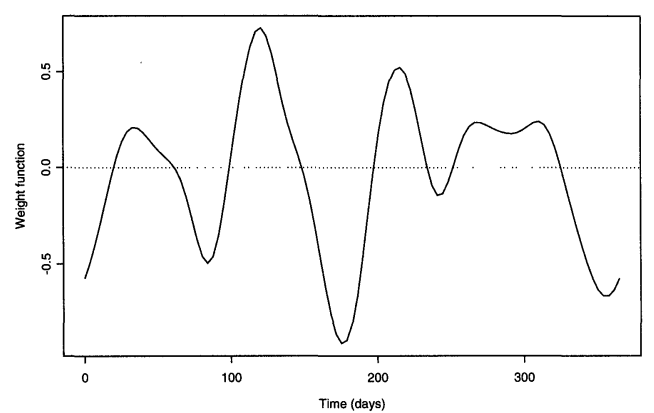

(a)

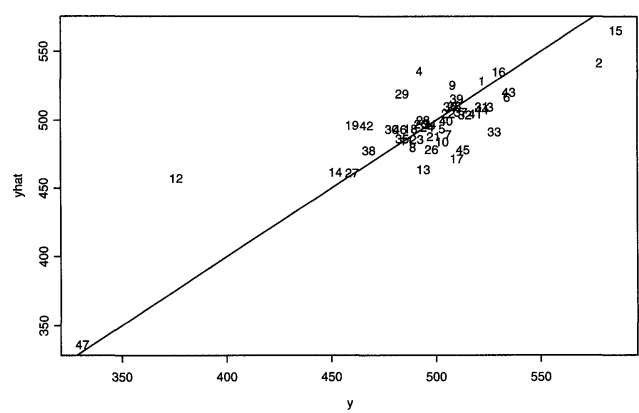

(b)

Fig. 4: Result of functional regression: (a) regression coefficient $\beta_{1}(s)$; (b) scatter plot of $y$ $(x$-axis) and $\hat{y}$ (y-axis)

scatter plot is given in Figure 6(b) to be used for the search for the reasons of large Cook's $\mathrm{D}$ based on the SIF. No.12 has large values of $\sqrt{h_{i i}} \times\left|r_{i}\right|$, and No.4 has large values of both of $\sqrt{h_{i i}} \times\left|r_{i}\right|$ and $p_{i i}$. The values of $h_{i i}$ and $\left|r_{i}\right|$ are given in Figure 5(b). Clearly the reasons of large $\sqrt{h_{i i}} \times\left|r_{i}\right|$ are the same as described above as the reasons for large Cook's D values based on the EIF. Table 1 gives the values of $\left|r_{i}\right|, h_{i i}$ and $p_{i i}$ of No. 4, 12, 2 (Aomori) and 47 (Okinawa) which give the largest four values of Cook's Ds based on the EIF and SIF. For detecting influential subsets of observations we apply the PCA of metric $\operatorname{Cov}\left(\hat{\mathbf{b}}^{*}\right)^{-}$to the dataset of EIF. The first four PCs explain $54.2 \%$ of the total variation. The scatter plots of $\mathrm{PC} 2$ vs. $\mathrm{PC} 1$ and $\mathrm{PC} 4$ vs. PC3 are shown in Figures 9(a) and 9(b). These figures suggest that there are two observations, No. 4 and 12, which may be singly influential, and some subsets such as $(29,42),(17,33)$ and $(45,13)$, which may be jointly influential. 
Sensitivity Analysis in Functional Regression Models

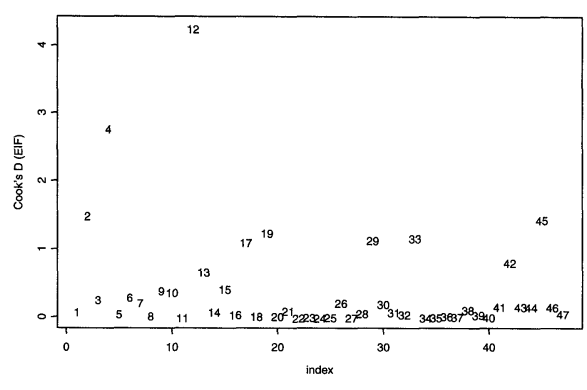

(a)

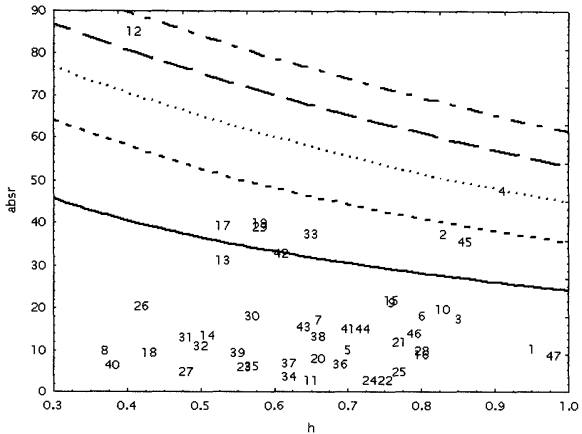

(b)

Fig. 5: (a) Cook's D based on EIF, (b) scatter plot of $h_{i i}$ (x-axis) and $\left|r_{i}\right|$ (y-axis)

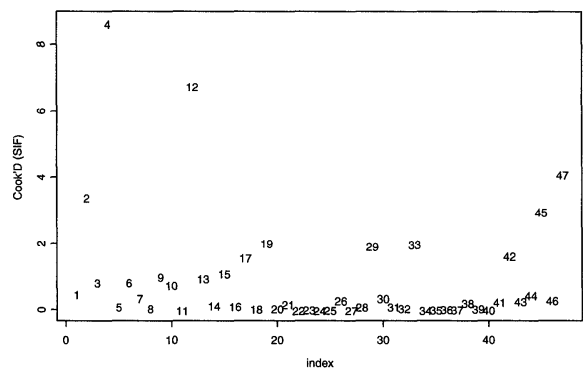

(a)

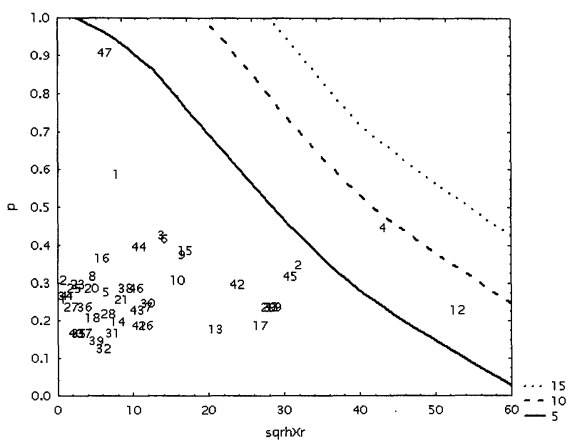

(b)

Fig. 6: (a) Cook's D based on SIF, (b) scatter plot of $\sqrt{h_{i i}} \times\left|r_{i}\right|$ (x-axis) and $p_{i i}$ (y-axis)

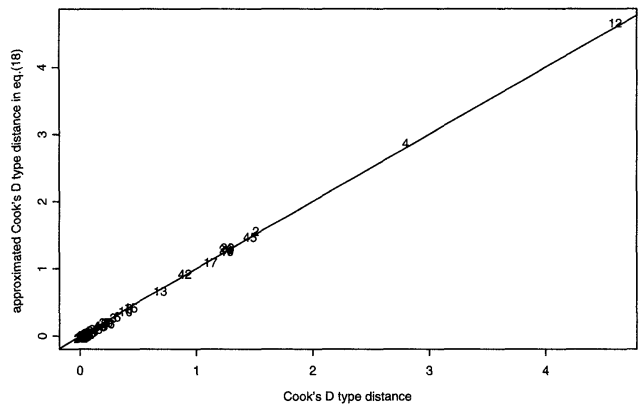

Fig. 7: $x$-axis: Cook's D defined by (8), $y$-axis: approximate Cook's D defined by (9) 
Table 1: Selected case statistics

\begin{tabular}{|c|c||c|c|c|c|c|}
\hline$i$ & location & $\left|r_{i}\right|$ & $h_{i i}$ & $p_{i i}$ & $D_{E P i}$ & $D_{S P i}$ \\
\hline \hline 2 & Aomori & 35.03 & 0.83 & 0.32 & 1.504 & 3.408 \\
\hline 4 & Iwate & 45.17 & 0.91 & 0.42 & 2.781 & 8.622 \\
\hline 12 & Tokyo & 82.71 & 0.41 & 0.20 & 4.258 & 6.772 \\
\hline 47 & Okinawa & 6.45 & 0.98 & 0.88 & 0.061 & 4.110 \\
\hline
\end{tabular}

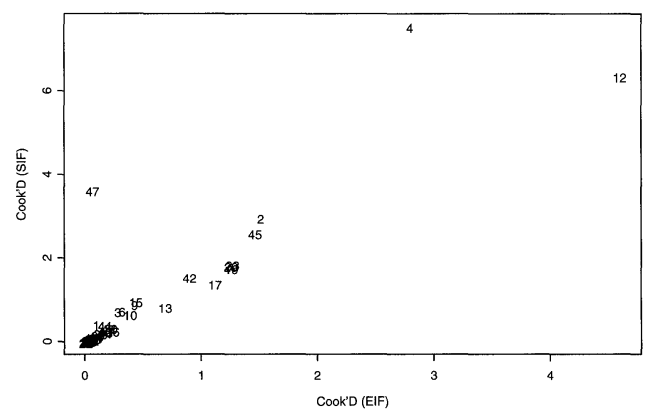

Fig. 8: Scatter plot of Cook's D (EIF) ( $x$-axis) and Cook's D (SIF) ( $y$-axis)

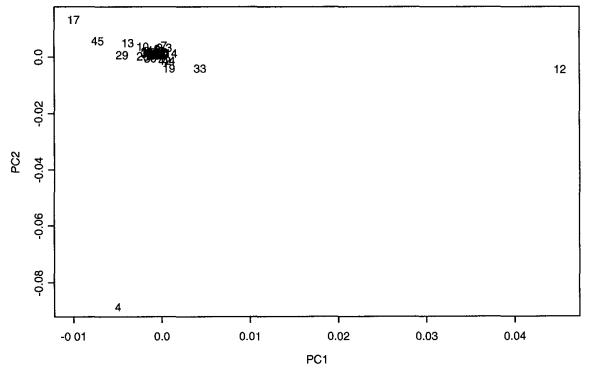

(a)

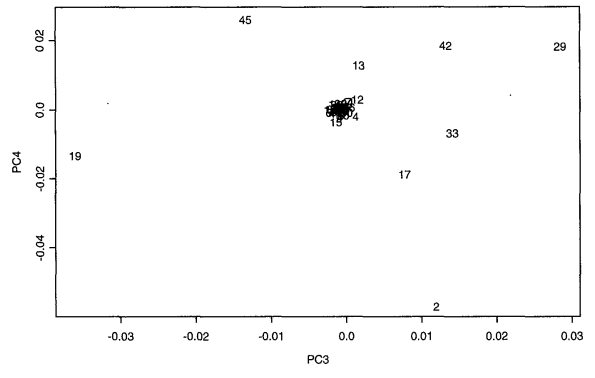

(b)

Fig. 9: Scatter plot: (a) PC1 and PC2; (b) PC3 and PC4

\section{Concluding remarks}

A method of sensitivity analysis is proposed for detecting influential observations in functional regression models. As basic tools empirical influence functions (EIF) and sample influence functions (SIF) are derived for regression coefficient functions in functional formulation and for coefficient vectors in matrix formulation using basis function expansions, and Cook's D type distance is defined both on the basis of the EIF and on the SIF.

Cook's D based on the EIF can be approximated by a product of leverage for non- 


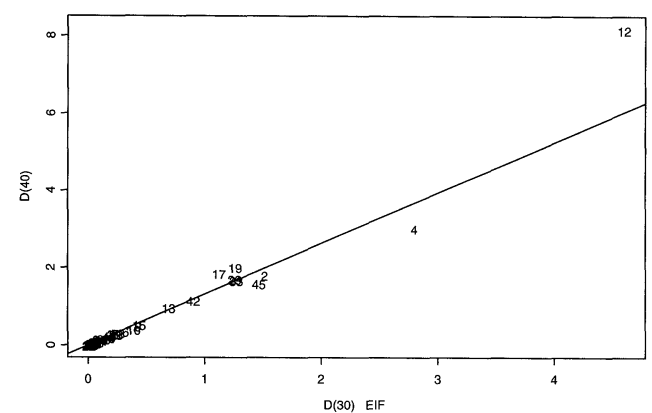

(a)

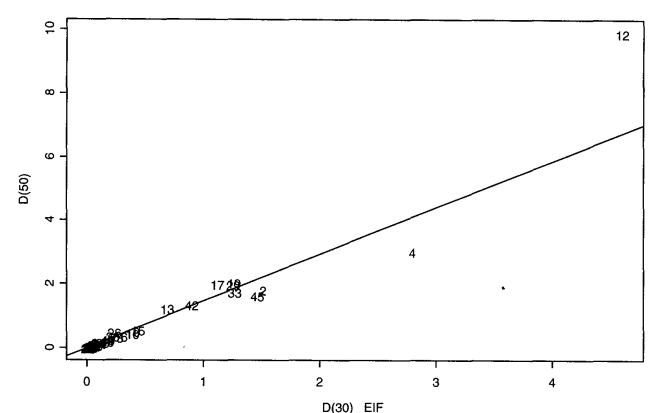

(b)

Fig. 10: Scatter plot of Cook's D based on EIF at $\lambda=10^{6}$ : (a) $x$-axis: $\mathbf{K}=30, y$-axis: $\mathbf{K}=40$, line: $y=\frac{42}{32} x$; (b) $x$-axis: $\mathbf{K}=30, y$-axis: $\mathbf{K}=50$, line: $y=\frac{47}{32} x$ $(32=\operatorname{rank}(\mathbf{Z})$ for $\mathbf{K}=30,42=\operatorname{rank}(\mathbf{Z})$ for $\mathbf{K}=40$ and $47=\operatorname{rank}(\mathbf{Z})$ for $\mathbf{K}=50$ )

penalized model $h_{i i}$ and residual $r_{i}$ if the second term and third term of (8) are sufficiently small. In our numerical example the approximation is very good as shown in Figure 7 . This approximate formula is very similar to the formula which holds between the Cook's D and (leverage, residual) in the ORA.

The ratio of Cook's Ds based on the EIF and SIF is equal to (1-leverage) ${ }^{2}$. This relation is also the same as with the case of the ORA, though the definition of leverage is different. Therefore, as in ordinary regression analysis Cook's D based on EIF sometimes does not provide a good approximation to that based on SIF in functional regression analysis because of the effects of leverage for the penalized model $p_{i i}$.

Looking at Figure 8 the discrepancy between Cook's D based on the EIF and the SIF is extremely large for No. 47 , for which both $h_{i i}$ and $p_{i i}$ are very large. In such cases the EIF does not provide a good approximation for the SIF as suggested by the equation (10).

To determine the value of smoothing parameter $\lambda$ and the number of basis functions $\mathbf{K}$, we used cross-validation method. For $\lambda$ we could find the optimum value in the sense that it gives the minimum cross-validated value. For the number of basis functions $\mathbf{K}$, however, it is noted that, as $\mathbf{K}$ increases, the cross-validated value decreases until it reaches the bottom and then it stays there. This fact suggests that we need not search for the optimal $\mathbf{K}$, if we use sufficiently large $\mathbf{K}$, and that the problem of determining the optimal $\lambda$ and $\mathbf{K}$ reduces to the problem of determining only the optimum value of $\lambda$. It coincides well with the description "in practice, values of $\mathbf{K}$ around 20 or 30 give good results" (Ramsay and Silverman, 1997; p.170).

However, there remains the question whether influence differs or not for different values of $\mathbf{K} \geq 30$. Figure 10 and 11 show the scatter plots of Cook's D at $\mathbf{K}=30$ vs. $40, \mathbf{K}=30$ vs. 50 , where each $\operatorname{rank}(\mathbf{Z})$ at $\mathbf{K}=30,40$ and 50 is 32,42 and 47 respectively. It is noted from these figures that the orders of the amount of influence are rather stable and that the Cook's Ds divided by $\operatorname{rank}(\mathbf{Z})=\sum_{i} h_{i i}$ take similar values for $\mathbf{K}=30,40$ and 50 .

In our formulation of deriving influence functions we consider that the smoothing parameter $\lambda$ is a constant. We may consider that $\lambda$ is variable and there may be indirect effects on the parameter estimates through the effect on $\lambda$. We neglect such effects because they 


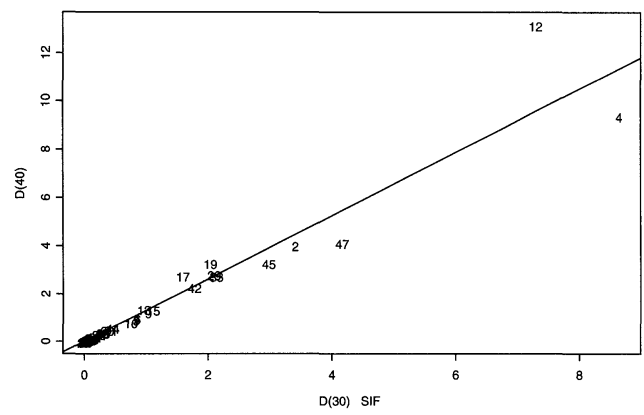

(a)

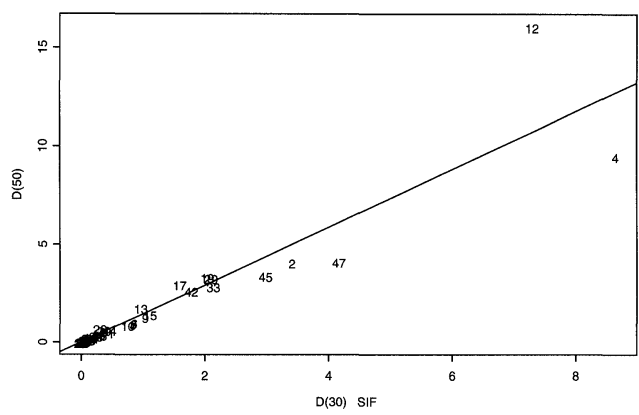

(b)

Fig. 11: Scatter plot of Cook's D based on SIF at $\lambda=10^{6}$ : (a) $x$-axis: $\mathbf{K}=30, y$-axis: $\mathbf{K}=40$, line: $y=\frac{42}{32} x$; (b) $x$-axis: $\mathbf{K}=30, y$-axis: $\mathbf{K}=50$, line: $y=\frac{47}{32} x$ $(32=\operatorname{rank}(\mathbf{Z})$ for $\mathbf{K}=30,42=\operatorname{rank}(\mathbf{Z})$ for $\mathbf{K}=40$ and $47=\operatorname{rank}(\mathbf{Z})$ for $\mathbf{K}=50)$

are small in sensitivity analysis in functional PCA (Yamanishi and Tanaka, 2005).

Also in our numerical example, the values of both Cook's D based on the EIF and the SIF change little for small variation of the smoothing parameter and the number of basis functions. To consider such indirect effects precisely will be part of our future work.

\section{Acknowledgements}

The authors are grateful to the anonymous referees for valuable comments and suggestions, which are helpful to improve the presentation of the paper.

This research was partly supported by Japan Society of Promotion of Science (Grantin-Aid for Scientific Research (C)(2)16500175).

\section{REFERENCES}

Ando, T., Imoto, S. and Konishi, T. (2001). Estimating nonlinear regression models based on radial basis function networks. Japanese Journal of Applied Statistics 30, 19-35. (in Japanese)

Box, G. E. P. and Jenkins, G. M. (1970). Time Series Analysis Forecasting and Control. San Francisco: Holden-Day.

Buja, A., Hastie, T. and Tibshirani, R. (1989). Linear smoothers and additive models (with discussion). Annals of Statistics 17, 453-555.

Chatterjee, S. and Hadi A. S. (1988). Sensitivity Analysis in Linear Regression. New York: John Wiley \& Sons.

Cook, R. D. and Weisberg, S. (1982). Residuals and Influence in Regression. London: Chapman and Hall.

Green, P. J. and Silverman, B. W. (1994). Nonparametric Regression and Generalized Linear Model: A Roughness Penalty Approach. London: Chapman and Hall.

Hampel, F. R. (1974). The influence curve and its role in robust estimation. Journal of American Statistical Association 69, 383-393. 
Harasawa, N., Fueda, K. and Tanaka, Y. (2004). Influence Diagnostics in Functional Regression Analysis for Scalar Responses. Technical Report No.89, Okayama Statistical Association. (in Japanese)

Japan Meteorological Agency (1999). Annual Report of Automated Meteorological Data Acquisition System $(A M e D A S)$. Tokyo: Japan Meteorological Business Support Center. (CD-ROM)

Pringle, A. A. and Rayner, R. M. (1971). Generalized Inverse Matrices: With Applications to Statistics (Griffin's Statistical Monographs and Courses, No. 28). London: Lubrecht \& Cramer Ltd.

Ramsay, J. O. (1982). When the data are functions. Psychometrika 47, 379-396.

Ramsay, J. O. and Dalzell, C. (1991). Some tools for functional data analysis (with discussion). Journal of Royal Statistical Society, Series B 53, 539-572.

Ramsay, J. O. and Silverman, B. W. (1997). Functional Data Analysis. New York: SpringerVerlag.

Shimokawa, M., Mizuta, M. and Sato, Y. (2000). An expansion of functional regression analysis. Journal of the Japanese Applied Statistics 29, 27-39. (in Japanese)

Simonoff, J. S. (1996). Smoothing Methods in Statistics. New York: Springer-Verlag.

Tanaka, Y. (1994). Recent advance in sensitivity analysis in multivariate statistical methods. Journal of the Japanese Society of Computational Statistics 7, 1-25.

Tanaka, Y. and Zhang, F. (1999). R-mode and Q-mode influence analyses in statistical modelling: Relationship between influence function approach and local influence approach. Computational Statistics and Data Analysis 32, 197-218.

Yamanishi, Y. and Tanaka, Y. (2005). Sensitivity analysis in functional principal component analysis. Computational Statistics 20, 311-326.

(Received January 2005, Accepted October 2005) 